\title{
Long-term simulations of extragalactic jets: cavities and feedback
}

\author{
Manel Perucho, Vicent Quilis and José María Martí \\ Departament d'Astronomia i Astrofísica, Universitat de València \\ Burjassot 46293 \\ email: manel.perucho@uv.es, vicent.quilis@uv.es, jose-maria.marti@uv.es
}

\begin{abstract}
We present long-term numerical simulations of powerful extragalactic relativistic jets in two dimensions. The jets are injected in a realistic atmosphere with powers $10^{44}, 10^{45}$ and $10^{46} \mathrm{erg} / \mathrm{s}$, during tens of Myrs. After this time, the jet injection is switched off. We follow the evolution of the jets and associated shocks from $1 \mathrm{kpc}$ to hundreds of kiloparsecs during more than 100 Myrs. The $10^{45} \mathrm{erg} / \mathrm{s}$ jet was simulated with leptonic and baryonic composition. Our results show that, for powerful jets, the main heating mechanisms are the driving shock-wave and mixing. We discuss the implications that these results have in the frame of cooling flows in clusters.
\end{abstract}

Keywords. galaxies:jets, galaxies: cooling flows, galaxies: active, relativistic hydrodynamics

\section{Introduction}

The rates of cooling of the cluster medium among the galaxies should result in the fall of this gas onto the galaxies, due to the energy loss of this gas (Fabian 1994). This should occur via the so called cooling flows, with mass-fall rates of up to $1000 M_{\odot} /$ yr. However, these flows are not observed commonly. The present paradigm for explaining this fact relies on heating by Active Galactic Nuclei (see McNamara \& Nulsen 2007 for a recent review on the topic). There has been debate about the way in which the heating may occur and whether it is possible at all that the energy brought by AGN jets from the core of the active galaxies to the cluster medium is enough to substantially reduce the cooling flow. The processes that have been invoked are heating by the bow-shock produced at the head of the jet, sound waves, turbulent heating in the wake of buoyant cavities or heat conduction, among others. Cavities have been observed in a number of clusters, despite the difficulty in the detection, as deficits in X-ray emission, normally filled by radio-emission from jet lobes in AGN. These cavities are thought to be close to pressure equilibrium with their environments because any bright rims or shells surrounding them appear to be cooler than the ambient, implying that they are not shocks.

Turbulent heating of the ambient in the wake of the buoyant cavities may become important when the pressure jump between the shocked material and the ambient becomes small. The assumption that buoyancy is the dominant cause of cavity motion comes from the idea that radio lobes reach pressure balance with the surrounding medium in a relative short time. After equilibrium is reached, the region inside the shocked ambient medium, including the shocked jet material and some mixed shocked ambient through the contact discontinuity, i.e., the cocoon, would turn into an X-ray cavity. Buoyancy, and further mixing favoured by smaller velocity gradients through the contact discontinuty would then be possible. In the last years shocks in powerful FRII jets have been reported, like those in Hercules A (Nulsen et al. 2005), Hydra A (Simionescu et al. 2009) or MS0735.6+7421 (McNamara et al. 2005) at distances of hundreds of kiloparsecs to 
the active nucleus. Some numerical studies have focused on the evolution of cavities by buoyancy (see, e.g., Quilis et al. 2001, Brüggen et al. 2009). Different works have addressed the problem from the evolution of jets, simulated as an injection of energy and momentum in central cells (Omma et al. 2004, Vernaleo \& Reynolds 2007, O'Neill \& Jones 2010), related to the accretion of cooling flows (Brighenti \& Mathews 2006, Cattaneo \& Teyssier 2007), or directly injected in one boundary (Zanni et al. 2005, Vernaleo \& Reynolds 2006). These works have considered intermittency in the galactic activity and some included pressure and density profiles. Most of them report successful transfer of injected energy to the ambient particles, but Vernaleo \& Reynolds (2006), who find a failure of the injected jets to stop the cooling flows. It is our aim to test this scenario via numerical simulations of relativistic jets evolving in a realistic environment from a close distance to the nucleus, $1 \mathrm{kpc}$, to hundreds of kiloparsecs, with the largest possible resolution. We present two-dimensional axisymmetric, RHD simulations following the evolution of jets with different properties, in which the injection of particles and energy at the inlet is gradually stopped after tens of Myrs.

\section{Simulations}

We used the finite-volume code Ratpenat, which solves the equations of relativistic hydrodynamics in conservation form using high-resolution-shock-capturing methods. Ratpenat was parallelized with a hybrid scheme with both parallel processes (MPI) and parallel threads (OpenMP) inside each process (see Perucho et al. 2010). The code also solves the equation of state of relativistic gas with two populations of particles, namely leptons and baryons.

The jets are injected in a grid including an ambient composed by neutral hydrogen with a King-like density profile in hydrostatic equilibrium. This profile has been taken from the fit to X-ray data of 3C 31 by Hardcastle et al. (2002) as typical for an elliptical galaxy. The density at $1 \mathrm{kpc}$ from the galactic nucleus is $0.1 \mathrm{~m}_{p} / \mathrm{cm}^{3}$. The corresponding dark-matter distribution forcing this equilibrium consists of a halo of $10^{14} M_{\odot}$ within $1 \mathrm{Mpc}$. We present here results from 4 simulations (see Table 1 for specific properties of each jet). The resolution depends on the simulation with cell size ranging from $50 \times 50$ parsecs $(\mathrm{J} 1)$ to $100 \times 100$ parsecs $(\mathrm{J} 2, \mathrm{~J} 3, \mathrm{~J} 4)$. The jets are injected at $1 \mathrm{kpc}$, with radius of $100 \mathrm{pc}$, i.e., one or two cells depending on resolution in a grid that is extended axially and radially as the bow-shock grows. In the radial direction, a grid with the given resolution extends up to $50 \mathrm{kpc}(\mathrm{J} 1)$ or $100 \mathrm{kpc}(\mathrm{J} 2, \mathrm{~J} 3, \mathrm{~J} 4)$. Then a grid with increasing cell size is added up to $100 \mathrm{kpc}(\mathrm{J} 1)$ or $150 \mathrm{kpc}(\mathrm{J} 2, \mathrm{~J} 3, \mathrm{~J} 4)$. Beyond this distance, the radial cell size is then kept constant at its maximum and the grid increased following the evolution of the bow-shock. Reflection boundary conditions are set at the basis of the grid to mimick the effect of a counter-jet. With the given grid and jet properties, the time-step during the first part of the simulations is of 50 to 100 years. The total injected energy ranges from a $3 \times 10^{59}$ to $10^{61} \mathrm{erg}$, depending on the simulation.

The injection of energy and particles through the jet is stopped after 50 Myrs (J1, J3, J4) or 16 Myrs (J2). Before this moment, the dynamics of the jets are dominated by the injection, following expected evolutions. During and after the switch-off, the decrease in the velocity of the head is immediate due to the short time-scales needed by the relativistic fluid in the jet to reach the terminal shock. Mach numbers of the bow-shocks fall from around 10 to values between 1 and 2, but remain in these values from the switch-off to times close to 200 Myrs in J1, J2 and J4. This represents a factor 3 of the active phase in the case of $\mathrm{J} 1$ and $\mathrm{J} 4$, and more than a factor 10 for $\mathrm{J} 2$, the most powerful jet. In all the simulations, the aspect ratio of the bow-shock approaches sphericity after 
Table 1. Parameters of the simulated jets. Column 1 gives the model, column 2 the injection velocity, column 3 the injection density, column 4 the leptonic number, column 5 the jet power, column 6 the maximum resolution, and column 7 the switch-off time.

\begin{tabular}{lcccccc}
\hline Model & Velocity $[c]$ & Density $\left[\mathrm{kg} / \mathrm{m}^{3}\right]$ & $\mathbf{X}_{e}$ & $\mathbf{L}_{k}[\mathrm{erg} / \mathrm{s}]$ & $\mathbf{m a x}$. resol. $[\mathrm{pc} /$ cell $]$ & $\mathbf{t}_{\text {off }}[\mathrm{Myrs}]$ \\
\hline $\mathrm{J} 1$ & 0.9 & $8.3 \times 10^{-26}$ & 1.0 & $10^{45}$ & 50 & 50 \\
$\mathrm{~J} 2$ & 0.984 & $8.3 \times 10^{-26}$ & 1.0 & $10^{46}$ & 100 & 16 \\
$\mathrm{~J} 3$ & 0.9 & $8.3 \times 10^{-27}$ & 1.0 & $10^{44}$ & 100 & 50 \\
$\mathrm{~J} 4$ & 0.9 & $8.3 \times 10^{-26}$ & 0.5 & $10^{45}$ & 100 & 50 \\
\hline
\end{tabular}

the end of the active phase. The jet material mixes completely with the shocked ambient, forming a low-density region, precursor of the buoyant cavity (see Fig. 1). Although this process starts before the switch-off, it is enhanced after this time. The calculation of the integrated X-ray luminosity across a 3D-box, produced from the axisymmetric grid, shows that this region already appears as less bright than the surroundings. None of our simulations has reached the stage of cavity buoyancy, as the pressure jump persists.

This mixed region is, at the late stages, formed by shocked ambient with polution of jet particles, with maxima of around two per cent of the latter in a given cell, implying very efficient mixing. Croston et al. 2008 report that radio lobes of low-power radiogalaxies present lower pressure than expected if it is obtained from the emission of hot particles. They suggest that the missing pressure is provided by entrained ambient particles, what could be confirmed by our results.

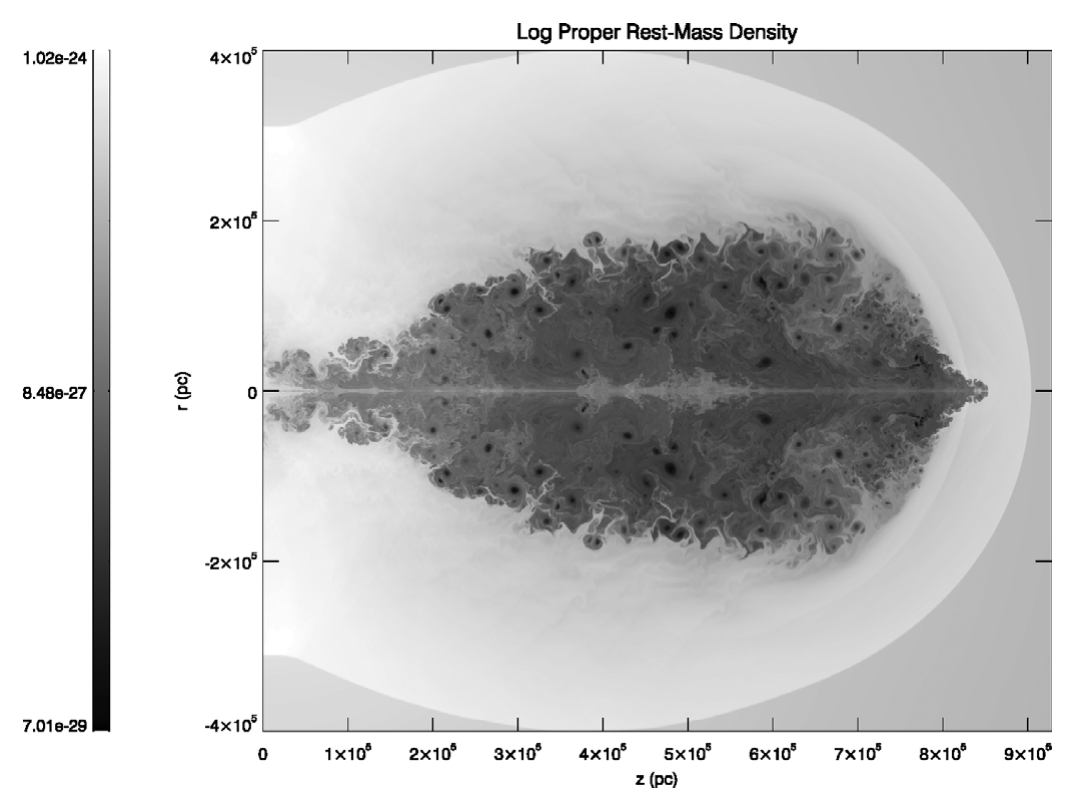

Figure 1. Maps of rest-mass density of simulation J2 at time 1.8 Myrs. The units are $\mathrm{kg} / \mathrm{m}^{3}$.

The energetic balance shows that around $95 \%$ of the injected energy goes to the ambient medium through shock-heating, mixing and acceleration. The rest iskept by the 
jet particles, invested into the gain of potential energy or lost in radiation. The amount of mass of the ambient that undergoes shock heating ranges from $10^{11}$ to $10^{12} M_{\odot}$ depending on the power injected during the active phase, in agreement with observational results (see McNamara \& Nulsen 2007). From this mass, typically 0.1 to $1.0 \%$ is mixed by instabilities arising in the contact discontinuity between the shocked ambient and the shocked jet fluid.

\section{Conclusions}

Our simulations show that the injection of a collimated, relativistic flow in the galactic and intra-cluster medium brings a large amount of energy to this gas, mainly through shock-heating and turbulent mixing with the injected, hot jet material. Contrary to what previously assumed, it seems that the shocks are persistent even during times longer than three times the duration of the active phase, and up to ten times in the case of J2. This result shows that buoyant cavities could represent the fate of radio-galactic relics, but are not needed in the process of stopping cooling flows. The amount of displaced and entrained gas is consistent with observations. In addition, we show that entrainment is very important and this could give an answer to reported missing pressure in radio-lobes if only hot particles are taken into account.

\section{Acknowledgements}

The authors want to acknowledge the use of supercomputational resources from the Spannish Supercomputational Network (RES), technical support and assistance from the staff of the nodes "Tirant", "Mare Nostrum" and "Magerit". The authors acknowledge financial support from the Spannish "Ministerio de Ciencia e Innovación" (MICINN) grants AYA2010-21322-C03-01, AYA2010-21097-C03-01 and CONSOLIDER2007-00050, and from the "Generalitat Valenciana" grant "PROMETEO-2009-103". MP acknowledges support from MICINN through a "Juan de la Cierva" contract.

\section{References}

Brighenti, F. \& Mathews, W. G., 2006, ApJ, 643, 120

Brg̈gen, M., Scannapieco, E., \& Heinz, S., 2009, MNRAS, 395, 2210

Cattaneo, A. \& Teyssier, R., 2007, MNRAS, 376, 1547

Croston, J. H., Kraft R. P., \& Hardcastle M. J., 2007, ApJ, 660, 191

Croston, J. H. et al., 2008, MNRAS, 386, 1709

Fabian, A. C., 1994, ARAA, 32, 277

Hardcastle, M. J. et al., 2002, MNRAS, 334, 182

Kraft, R. P., Nulsen, P. E. J., \& Birkinshaw, M., 2007, ApJ, 665, 1129

McNamara, B. R. \& Nulsen, P. E. J. 2007, ARAA, 45, 117

McNamara, B. R., Nulsen, P. E. J., Wise, M. W. et al., 2005, Nature, 433, 45

Nulsen, P. E. J., Hambrick, D. C., McNamara, B. R. et al., 2005, ApJ (Letters), 625, 9

Omma, H., Binney, J., Bryan, G., \& Slyz, A., 2004, MNRAS, 348, 1105

O'Neill, S. M. \& Jones, T. W., 2010, ApJ, 710, 180

Perucho, M. \& Martí, J. M., 2007, MNRAS, 382, 526

Perucho, M., Martí, J. M., Cela, J. M., Hanasz, M., de la Cruz, R., \& Rubio, F., 2010, A\&A, 519, A41

Quilis, V., Bower, R. G., \& Balogh, M. L., 2001, MNRAS, 328, 1091

Simionescu, A., Roediger, E., Nulsen, P. E. J. et al., 2009, A\& $A, 495,721$

Vernaleo, J. C. \& Reyynolds, C. S., 2006, ApJ, 645, 83

Vernaleo, J. C. \& Reyynolds, C. S., 2007, ApJ, 671, 171

Zanni, C., Murante, G., Bodo, G., Massaglia, S., Rossi, P., \& Ferrari, A., 2005, A $\& A$ A 429, 399 


\section{Discussion}

YUAN: The jet is well collimated, but the observed cavity is spherical-like. Some people argue we must require a B-field.

Perucho: I disagree. Observations seem to indicate that the lobes are dominated by thermal gas. In our simulations, the cavities form after the jets are switched off, without any need of magnetic fields. 